\title{
eCollaboration: On the nature and emergence of communication and collaboration technologies
}

\author{
Kai Riemer • Charles Steinfield • Doug Vogel
}

Published online: 12 October 2009

(C) Institute of Information Management, University of St. Gallen 2009

\section{Introduction}

The past decades yielded fundamental changes to the ways in which value creation is being organized. Globalization and market deregulation motivate businesses to expand into new, often remote markets, which requires them to decentralize and distribute work and often to partner with local organizations, which in turn brings with it changes to the ways in which people need to organize their work (Bleecker 1994). Also, shorter product life-cycles and the pressure to constantly innovate have led many businesses to experiment with new organizational structures (Quinn 1992). This leads to a situation, in which businesses today need to manage numerous external collaborative relationships with other parties in the marketplace (Ebers 1999). Furthermore, traditional internal structures are being broken up in order to give way to more flexible and responsive work setups. The ramifications of these developments are that people increasingly work in dispersed setups and are involved in changing, multi-project setups with others from different organizational backgrounds and geographical sites

\author{
K. Riemer $(\bowtie)$ \\ The University of Sydney, \\ Sydney, Australia \\ e-mail: k.riemer@econ.usyd.edu.au \\ K. Riemer \\ Universität Münster, \\ Münster, Germany \\ C. Steinfield \\ Michigan State University, \\ East Lansing, Michigan \\ D. Vogel \\ City University of Hong Kong, \\ Kowloon, Hong Kong
}

(Bélanger et al. 2003; Lipnack and Stamps 2000; Weinkauf et al. 2004). Hence, since people often do not share the same organizational and physical environments with their direct work associates, they have to rely on technology to create shared virtual work environments.

In the above context, eCollaboration as a topic refers to all issues of ICT-based collaborations within and between organizations. More specifically, the term eCollaboration describes practices of communication, coordination and collaboration between people in distributed contexts, such as projects, (virtual) teams, or processes in and between organizations, which are enabled by Information and Collaboration Technology, in essence: eCollaboration systems. Alternative terms exist to describe this class of ICT, such as: Groupware system, CSCW system, Workgroup Computing, Collaborative Software or Cooperation systems.

eCollaboration systems have come a long way since the first experimental systems were piloted and spearheaded by the Computer-Supported Collaborative Work (CSCW) research community (Greif 1988; Schmidt 1991; Teufel 1996; Wendel 1996). Today, fuelled by advances in software and in particular Internet technology (e.g. Web 2.0 interface techniques) many new systems and technologies have emerged. However, while technology vendors stress the new possibilities offered by their products for managing collaborations, the realities in distributed projects, virtual teams, and inter-organizational networks quite often paint a different picture. E-Collaboration is a complex, precarious, and too often rather ineffective undertaking. While the resulting (virtual) work environments present manifold managerial challenges, at the same time the respective eCollaboration technologies are still emerging at a fast pace. Computer software and telecommunications technology are increasingly converging to create new integrated eCollaboration platforms, while the 
developments in Internet technology and use thereof have yielded new social software-based collaboration opportunities. Hence, eCollaboration is a field in motion, technologically and managerial.

To this end, this focus theme issue has attracted a range of research and position papers, which study and discuss various facets of eCollaboration. The diversity of issues covered across the papers is testimony to the complexity and multi-faceted nature of the eCollaboration field. Before we give a brief overview of the papers included in this focus theme issue, we will provide an introductory overview to the eCollaboration field from a market perspective. Scholars have deplored the absence of the technological artifact in IT-related research (Orlikowski and Iacono 2001). Against this backdrop, the aim of this paper is to take a look at the eCollaboration artifacts and their emergence over the past years, in order to set the scene for the then following research papers. In doing so, its aim however is not to provide a comprehensive research overview of the domain.

We begin by reviewing market conditions and organizational developments that have both fuelled and are affected by the emergence of new collaborative technologies and eCollaboration as a phenomenon. In "Features and Building blocks" we introduce typical building blocks and features of eCollaboration systems, before we take a brief look at the emergence of eCollaboration technologies over the past three decades. In doing so, we discuss current trends that are likely to affect organizations in the upcoming years (in "Emergence and historical developments"). We then discuss the nature of eCollaboration systems as open platform technologies (in "Nature of eCollaboration systems as open systems") and briefly present implications for research and practice. Finally, we provide a brief structured overview of research in the eCollaboration field and position the papers included in this issue.

\section{Market developments and organizational changes as drivers}

Value creation is increasingly characterized by interorganizational division of labor and corresponding collaboration activities with various business partners. We identify three major market drivers responsible for the ongoing change processes: Firstly, globalization and the liberalization of markets lead organizations to venture into new (geographical) areas, which results in organizational structures that span national and geographic boundaries and spurs the emergence of multi-national alliances. Secondly, the increasing informatization and knowledge-intensity of products and processes means that virtually no company is able to develop and own all knowledge required for designing, marketing and providing its products or services (Ciborra 1992; Ireland et al. 2002; Prahalad and Hamel 1990). As a consequence, companies are increasingly teaming up with external partners for organizing innovation and knowledge creation processes (Powell 1987). Thirdly, the shorter lifecycle of products and the pressure to constantly innovate, in markets were customers increasingly expect to be provided with individualized services, drives businesses to experiment with new, virtualized forms of organizing and to outsource non-value adding processes (Franke 2001). Together, these market drivers lead to increases in inter-organizational collaborations and an ongoing reorganization and flexibilization of internal structures, both of which stimulate the emerging eCollaboration phenomenon.

The emergence of so-called hybrid forms of organization (Quinn 1992) is a reaction to the above-described market trends. In many industries for example, the role of suppliers is changing, from simple providers of goods to valuable partners that play important roles in process improvements and new product developments (Sheth and Sharma 1997). Moreover, many industries, such as the airline (e.g. StarAlliance, OneWorld) and electronics industries (e.g. SonyEricsson) have seen the emergence of large interorganizational business networks, in which eCollaboration technologies play an important role in the daily operations and management practices (Riemer and Klein 2006). Similarly, other heavily ICT-based organizational arrangements have emerged, such as virtual organizations (VOs), which aim to enable small and medium-sized players to gain virtual size for jointly producing and marketing their products and services (Franke 2001; Saabeel et al. 2002). Here, again, eCollaboration technologies are necessary to enable operations on the group or team level (Riemer and Vehring 2008).

In addition to increases in external collaborations, many organizations also have changed internally in order to become more flexible and reactive to external changes (Lau et al. 2001). For example, new concepts to office space sharing allow an increasingly mobile workforce to roam flexibly across geographical office sites, by allowing them to book desk space over the Internet (vgl. Schmalzl et al. 2004). Moreover, virtual teams have become an indispensible form of organizing (Lipnack and Stamps 2000), driven by both increases in geographic spread of large organizations and the flexible staffing of projects with freelance workers from outside of the organization (Lavin Colky et al. 2002). Again, as a consequence of increases in mobility and spatial distribution, eCollaboration becomes the backbone for coordinating and working in the resulting work environments. Figure 1 illustrates how market drivers and organizational trends fuel the emergence of eCollaboration. 
Fig. 1 Trends driving the emergence of eCollaboration

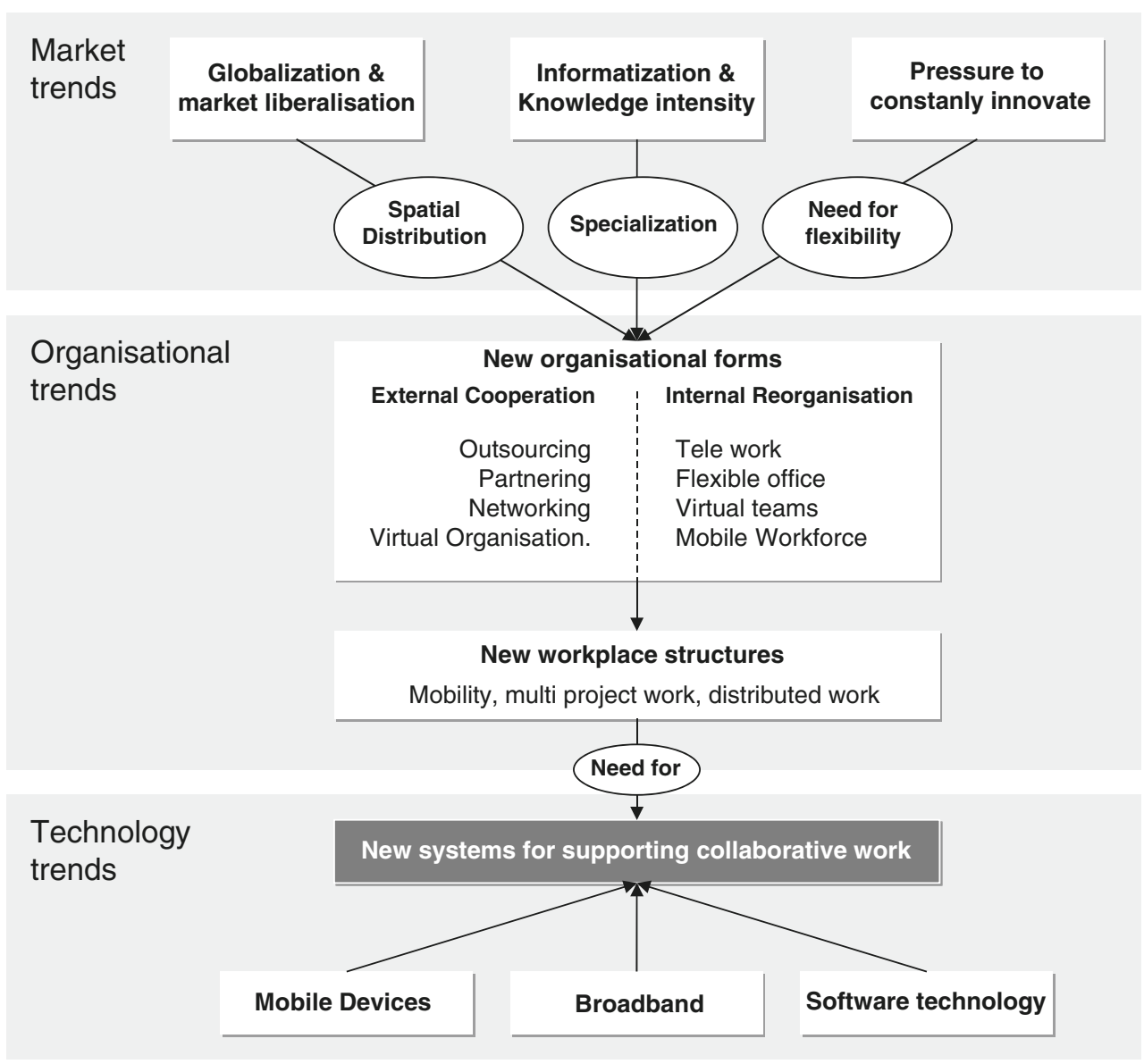

\section{Features and building blocks}

In order to develop an understanding of the eCollaboration phenomenon it is necessary to first of all take a closer look at the enabling technologies. eCollaboration systems support a wide range of inter-personal interactions, such as communication via a range of different media, the coordination of joint activities (e.g. tasks and processes), as well as the collaboration on joint objects (e.g. documents). It is worth noting that these three forms of interaction form a hierarchy (see Fig. 2), as coordination of group activities requires communication and collabora-

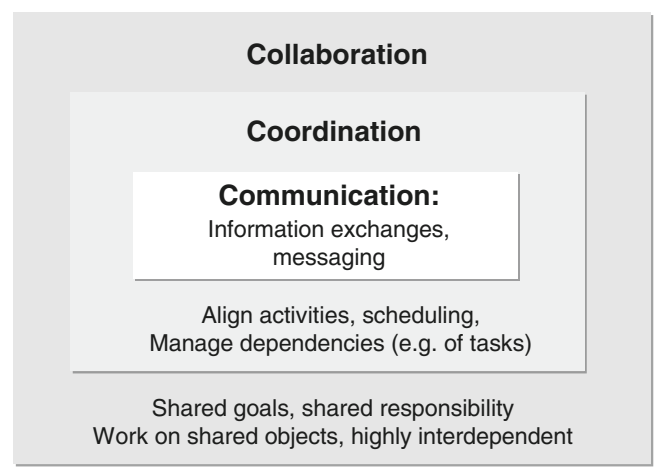

Fig. 2 Forms of interaction in group work (acc. to Teufel et al. 1995) tion on joint objects requires to coordinate access to this object. It also requires communication with regards to work progress, for example in order to negotiate changes and achieve consensus (Teufel et al. 1995). eCollaboration systems provide a multitude of features and building blocks, which can be classified according to these types of group interaction (see Table 1). In the marketplace, an abundance of dedicated tools exists, which support specific features, while more and more systems emerge, which resemble integrated platforms with a wide range of eCollaboration features (Riemer 2009).

\section{Communication media}

Communication is fundamental to any group work; it facilitates information exchange among group members (Wendel 1996) and is necessary for developing a shared group understanding (Wagner 1995). To this end, eCollaboration systems come equipped with different communication features in terms of different media such as text, audio, or video. Text-based communication for example comprises eMail, Instant Messaging, discussion boards, and newer forms such as Weblogs (Blogs). While some tools only support one form of communication, novel technologies such as Unified Communications infrastructures come 
Table 1 Examples of eCollaboration features and system building blocks

\begin{tabular}{lll}
\hline Communication & Coordination & Collaboration \\
\hline - eMail & - Group calendars & - Wikis \\
- Text chat & - Project management & - Group editors \\
- Instant Messaging & - Workflow management & - Electronic whiteboard \\
- Audio chat or conferences & - Social networking & - Application sharing \\
- Video chat or conferences & - Voting & - Shared Office solutions \\
- Discussion boards & - Document archives & \\
- Weblogs (Blogs) & - Version management & \\
\hline
\end{tabular}

with a range of integrated and coordinated communication media, such as audio, video conferencing, integrated with presence awareness technology (Riemer and Taing 2009).

\section{Coordination features}

Whenever people communicate in order to align their behavior in joint tasks or for accessing shared resources, be it time-wise, content-wise or in relation to other dependencies, we speak of coordination (Back and Seufert 2000). Coordination features subsume time coordination (e.g. scheduling group appointments), process coordination (e.g. workflow management) and project-related task coordination (e.g. project planning) (Schwabe 2001). Hence, common coordination features in eCollaboration systems are group calendars, task lists, project plans, and workflow planning and management. Moreover, social networking functionality can help in finding and allocating experts to group tasks, while sophisticated document spaces can help with filerelated coordination, such as versioning and coordinating joint document access (e.g. track changes), in order to avoid data loss and inconsistencies.

\section{Collaboration support}

Finally, collaboration is characterized by shared goals (working towards the same goal) and the work on shared objects, such as documents (Teufel et al. 1995). Consequently, some features of eCollaboration systems are dedicated towards enabling collaborative work, be it synchronous or asynchronous. For example, wikis can be used to enable joint authorship of a text document in asynchronous collaboration, while group editors create a synchronous work environment, in which each team member can see in real-time the text changes of other team members and participate in the creation of a joint document. Another form of synchronous collaboration is presented by so-called electronic whiteboards, which enable to draw and sketch in synchronous fashion. The most sophisticated real-time collaboration solutions are shared office solutions, which enable users to work together on standard office documents (e.g. spread sheets) and application sharing solutions, which allow users to share and jointly (and remotely) control the same computer screen, thereby working together with any form of computer software.

\section{Emergence and historical developments}

eCollaboration systems are not a new class of system, despite their recent popularity and increasing prevalence in organizations. In fact, the first systems for collaboration support appeared in the marketplace more than 20 years ago, driven by research and prototypes in the Computer Supported Cooperative Work (CSCW) research domain. The technical basis for ICT-based collaboration emerged with the advent of personal computers and widespread computer networks. However, it was the developments of the past decade, i.e. the spread of the Internet, recent advances in software technology, the emergence of IPbased speech technology and new mobile devices, which have paved the technological ground for today's eCollaboration phenomenon. In retrospect, it is possible to identify the following development phases.

Experiments: Prototypes und creative developments

The emergence of the CSCW research community in the 1980s marks the beginning of a phase of experimentation, which lasts until the early 1990s. This phase is characterized by design-oriented research, creative experimenting and development of prototypes in areas such as desktop video conferencing, workgroup computing or document management. However, diffusion of these systems into practice only happened slowly and on a fairly low level. The first eCollaboration technologies to show wider adoption are text-based communication technologies, most notably electronic blackboards or discussion boards and the first eMail systems (Connolly and Pemberton 1996).

Discovery: Diffusion of eMail und the emergence of groupware

The second phase, which we term 'discovery', spans the 1990s; it is characterized by a dynamic and widespread 
diffusion of eMail and the emergence of integrated groupware systems, such as Lotus Notes and Microsoft Exchange/ Outlook. These systems combine eMail functionality with (group) calendars, simple task management, and document coordination features. While eMail became an integral part of organizational work practices at the time, other collaborative systems fell well behind the predicted use (Buzzi di Marco 2000). eMail was found to account for about $95 \%$ of all collaborative interactions (Levitt and Mahowald 2002).

Diversification: internet as the main driver

The third phase we term diversification; it begins with the Dot.com boom. With Internet technology making fast inroads into organizations, software development paradigms changed and the Internet was more and more perceived as a software platform than a mere Information medium. A first wave of Internet-based software, termed application service provision (ASP), led to the development of many new and diverse Internet-based eCollaboration systems. This was matched by an increase in organizational demand for collaborative technologies, in large parts due to the above described market developments. These trends have led to the emergence of many new, often specialized eCollaboration systems, which at the same time led to a rather heterogeneous and unstructured market (Riemer 2009).

Integration und convergence: platform technologies

For a few years now parts of the market for eCollaboration systems show signs of consolidation and integration (Riemer 2009). There have been a range of mergers between systems providers and the systems as such also show signs of functional integration. This resulted in the emergence of large-scale eCollaboration platforms. Typical systems by well-known providers, such as IBM, Oracle, or Hyperwave today provide a broad range of eCollaboration features. Moreover, software vendors in neighboring markets began perceiving the eCollaboration market as a profitable area, with providers of document management systems, network services, databases or enterprise software integrating eCollaboration features into their own solutions or acquiring smaller vendors of collaborative software, in order to complement their portfolio (Gilbert et al. 2003). Most notably, there is a tendency towards integrating richer sets of communication media in eCollaboration systems (e.g. Lazar 2006). This trend is fuelled by the popularity of both Instant Messaging as well as voice-over-IP telephony. So-called real-time (or unified) collaboration platforms (e.g. IBM Sametime or Microsoft Office Communicator) integrate a diverse set of media and features such as unified messaging, instant messaging, IP telephony, presence management, as well as video conferencing (Lazar 2006; Riemer and Frößler 2007). These systems are the result of a convergence of desktop and enterprise software with telecommunications infrastructures (Riemer and Taing 2009).

Democratization: The age of social software

In parallel to the integration and convergence trend, another trend emerged recently fuelled by advances in software technology, most notably Internet user interfaces (e.g. Ajax and Web 2.0). The emergence of so-called social software, which is changing the ways in which the Internet is perceived and used, has considerable implications for the eCollaboration software market. Based on the principles of grass roots and user participation (user-generated content), such technologies mark the beginning of a democratization process of content generation on the web. Meanwhile, many organizations have found these principles applicable and useful to promote internal teamwork and knowledge sharing. Technologies like Wikis and Blogs have made successful inroads to the collaborative practices in organizations (e.g. Richter and Koch 2008). Wikis are being used for capturing team knowledge, Blogs provide a means of team information dissemination, social networking services allow for the identification of experts in large teams and provide means to propagate network awareness, while social tagging can be used to promote content-related group awareness.

All in all, the market for eCollaboration software currently experiences two more or less opposing trends. On the one hand, large integrated platforms emerge (e.g. Unified Communications), which exhibit a broad range of features and resemble infrastructure technologies more than ready-to-use systems, as they need customizing and proper organizational implementation. Consequently, these technologies lend themselves to a top-down implementation and management approach, as they require to be integrated with and linked to existing software (e.g. enterprise software) or physical infrastructures (such as the telephony network) (Riemer and Taing 2009). On the other hand, a plethora of small, often deliberately simple tools has emerged from the Web 2.0 space. Being based on the above-described principles, such technologies often make their way into organizations in a bottom-up manner, being driven by early adopters. Both types of technologies come with their own managerial and research challenges and require ongoing research efforts in order to better understand potentials and implications for users, managers and organizations. And while they might be different in terms of size, scope, technological complexity and underlying principles, they share the same characteristics as open systems. 


\section{Nature of eCollaboration systems as open systems}

While it is useful to view eCollaboration systems as bundles of features, which provide certain technical affordances (as we did in "Features and Building blocks"), it does not reflect the true nature of these technologies. We argue that an essential characteristic of eCollaboration technologies is a form of openness that can best be expressed in German language as Nutzungsoffenheit, whereby the artifact does not lend itself to or even determines a particular form of usage. Nutzungsoffenheit has a different quality as versatileness, with the latter describing that users can "modify and appropriate different parts of the [technology] in ways unforeseen by the technology designers." (Pipek and Wulf 2009, 6). However, Nutzungsoffenheit means that the true nature and potential of such technologies does only manifest when people make sense of and incorporate them in their day-to-day work routines. In essence, the technology and its set of features do not precipitate its forms of usage. As such, eCollaboration systems differ from other types of technologies. For examples, ERP systems have been described as inflexible and rigid, as they are deliberately designed to prescribe and control certain ways of working. In fact, organizational ('best practice') processes and the logic of carrying out tasks are inscribed in the technology by way of their design (Kallinikos 2004). Hence, these systems presuppose ways of carrying out work and thus impose their structures on the work practices of the users.

However, while the features provided by eCollaboration technologies give some direction for use (e.g. social networking, instant messaging etc.), these systems can essentially be applied for a wide range of activities, as they aim mainly at enabling interaction processes between people, which can be embedded in the context of rather diverse work practices. Studies have shown that seemingly simple tools such as Skype can enable highly diverse, situationally motivated communication practices (Riemer et al. 2007). In the contexts of these practices people draw on specific features, but ignore others, in enabling new or transforming existing practices. This in turn means that the adoption, appropriation and use of eCollaboration systems tend to be very situational.

In essence, eCollaboration technologies need to be understood as open and versatile technologies, in that they reveal and enfold their full potential only in the context of emerging practices of communication, coordination and collaboration. Consequently, such open technologies need to be interpreted and appropriated to become part of the work environment and of shared work practices. The actual properties of such technologies are not just those intended by their designers, but they are to a significant extend the product of user actions (Markus 2005), i.e. the appropria- tion and (re-) interpretation by its users. These observations have certain research implications. Future research needs to aim for a better understanding of the effects, implications, and ways of usage of such systems, as well as the factors that drive their adoption. Consequently, more empirical research is needed, which deals with these technologies in the context of use, i.e. in the workplaces of people in (virtual) teams, organizations, and social networks.

\section{Research perspectives and focus theme overview}

Owing to the multi-faceted nature of the eCollaboration phenomenon and its technologies, research in the domain can be equally diverse. In order to shed light on the nature of research included in this focus theme issue, we propose a set of dimension for classifying research efforts in the domain. We will briefly describe theses dimensions and characterize the research papers included in this focus theme issue.

First of all, eCollaboration research can be characterized by its level of analysis. While eCollaboration technologies generally operate on the team or community level, as they enable and support inter-personal interactions of various kinds, research can nevertheless take different perspectives and may span different levels of analysis. For example, research on systems design or adoption might focus on individual level factors such as usability and interaction design. Other research efforts might discuss the implications of eCollaboration in the larger context of organizations or even business networks or society as a whole. Secondly, eCollaboration research might focus on specific usage and application domains such as distributed software development, collaborative text writing or knowledge management. Thirdly, research can focus on certain stages of the systems life-cycle: it might be concerned with designing new technologies for supporting e-collaborative tasks, investigate the factors driving or inhibiting systems adoption or study the anatomy of systems appropriation. Moreover, studies might focus on eCollaboration work practices or the effects (on different levels of analysis) of certain eCollaboration technologies, or the management and use thereof. Finally, research in the domain can also focus on certain technologies (e.g. Skype) or otherwise decide to research the effects of a broader range of technologies. Figure 3 provides an overview of typical dimensions for classifying eCollaboration research, while Table 2 describes the research papers included in this issue.

The three research papers included in this focus theme issue are indicative of the breadth and diversity of eCollaboration research, as they cover different levels of analysis, life-cycle stages, usage domains, and technologies. The paper by Peter Rittgen provides a design-oriented 
Fig. 3 Typical dimensions for classifying eCollaboration research

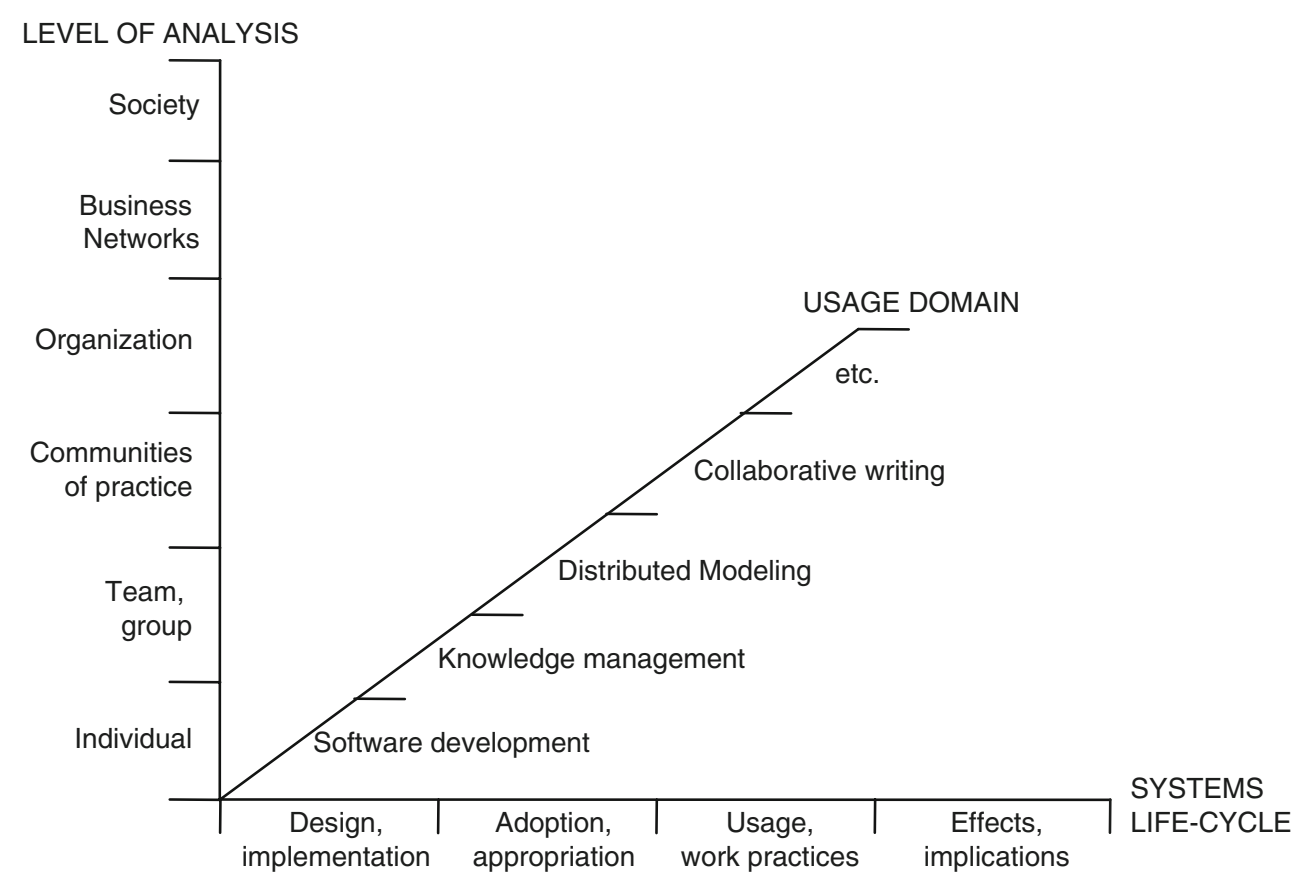

approach in that it investigates the usefulness of a particular tool designed for supporting distributed process modeling and negotiation tasks. As such the study operates on the group/team level and investigates the design of a tool and its subsequent use in an experimental research setup. The usage context is distributed process modeling and the technology is a domain specific tool, which supports complex collaboration tasks in an asynchronous fashion.

The second paper by Gang Peng and Peter Woodlock investigates the adoption of eCollaboration technologies in online social networks, which span organizational boundaries. Their study focuses on open source software development and researches a domain-specific coordination system (Subversion), which supports distributed work on software code. The study investigates the impact of network and recency effects on the adoption of Subversion by open source networks.

The third paper, written by Carolina Lopez-Nicolas and Angel Merono-Cerdan, investigates the role of eCollaboration technologies in the context of knowledge management. Their study operates on the organizational level in that it investigates the relationship between organizational culture (i.e. different types of culture) and the use of ICTs in the context of knowledge management. As such, the paper is concerned with organizational effects of eCollaboration technologies, depending on the type of culture exhibited by particular organizations.

Besides the three research papers, this focus theme issue also features two position papers. This paper format gives authors the opportunity to address contemporary topics, to apply fresh perspectives on existing phenomena and to take controversial positions. In his position paper, Ned Kock investigates the effects of oral speech support (or lack thereof) on the performance of e-collaborative tasks. In doing so, the author takes an evolutionary perspective and argues that oral speech is a costly trait evolved by our human ancestors to enable effective knowledge communication. By linking current research on group communications to human evolution the author offers a fresh perspective, which furthers our understanding of eCollaboration phenomena. In the second position paper, Niels Pinkwart and Hannes Olivier discuss the emerging phenomenon of cooperative virtual environments (CVEs), i.e. 3D virtual worlds such as Second Life, and their

Table 2 Overview of research papers included in this focus theme issue

\begin{tabular}{lllll}
\hline Paper & $\begin{array}{l}\text { Level of } \\
\text { analysis }\end{array}$ & $\begin{array}{l}\text { Systems life-cycle } \\
\text { phase }\end{array}$ & Usage context / domain & Technologies \\
\hline Rittgen & Team, work group & Design, usage & Distributed modeling & $\begin{array}{c}\text { Collaboration: Domain } \\
\text { specific tool } \\
\text { Coordination: Subversion }\end{array}$ \\
$\begin{array}{llll}\text { Peng/Woodlock } \\
\begin{array}{c}\text { Lopez-Nicholas/ } \\
\text { Merono-Cerdan }\end{array}\end{array}$ & Social network & Adoption & $\begin{array}{c}\text { Open source software } \\
\text { development } \\
\text { Knowledge Management }\end{array}$ & Diverse set of ICTs \\
\hline
\end{tabular}


role and implications for eCollaboration. The authors investigate whether or not CVEs are a lasting phenomenon that impacts on eCollaboration practice and research, or just an isolated episode within the online gaming community.

\section{References}

Back, A., \& Seufert, A. (2000). Computer Supported Cooperative Work (CSCW) - State-of-the-Art und zukünftige Herausforderungen. HMD-Praxis der Wirtschaftsinformatik, 37(213), 5-22.

Bélanger, F., Watson-Manheim, M.-B., \& Jordan, D. H. (2003). Aligning IS research and practice: a research agenda for virtual work. In M. Khosrow-Pour (Ed.), Advanced topics in information resources management (pp. 1-31). Hershey: Idea Group.

Bleecker, S. E. (1994). The virtual organization. The futurist, 28(2), 9-14.

Ciborra, C. U. (1992). Innovation, networks and organizational learning. In C. Antonelli (Ed.), The economics of information networks (pp. 91-102). Amsterdam.

Connolly, J. H., \& Pemberton, L. (1996). Linguistic concepts and methods in CSCW. Berlin et al.

Buzzi di Marco, G. (2000). Ein Konzept für das Design von CSCWSystemen zur Unterstützung von Kommunikation und Zusammenarbeit in Innovationsprojekten. Flein.

Ebers, M. (1999). Explaining inter-organizational network formation. In M. Ebers (Ed.), The formation of inter-organizational networks (pp. 3-40). Oxford: Oxford University Press.

Franke, U. (2001). The concept of virtual web organisations and its implications on changing market conditions. eJoV-Electronic Journal of Organizational Virtualness, 3(4), 43-64.

Gilbert, M. R., Shegda, K. M., Phifer, G., \& Knox, R. E. (2003). Content management, portals and collaboration converge. from www.gartner.com.

Greif, I. (ed). (1988). Computer-supported cooperative work: A book of readings. San Franscisco: Kaufmann.

Ireland, R. D., Hitt, M. A., \& Vaidyanath, D. (2002). Alliance management as a source of competitive advantage. Journal of Management, 28(3), 413-446.

Kallinikos, J. (2004). Deconstructing information packages: organizational and behavioural implications of large scale information systems. Information Technology and People, 17(1), 8-30.

Lau, T., Wong, Y. H., Chan, K. F., \& Law, M. (2001). Information technology and the work environment - does IT change the way people interact at work? Human Systems Management, 20(3), 267-279.

Lavin Colky, D., Colky, M. T., \& Young, W. H., III. (2002). Managing and developing people in the virtual organization. Malabar: Krieger.

Lazar, I. (2006). Integrating telephony, IM, video and mobility with presence. Business Communications Review, (June), 28-31.

Levitt, M., \& Mahowald, R. P. (2002). There should be more to collaboration than email. An IDC White Paper Retrieved 200409-15, from http://www.groove.net/pdf/idc-collaboration.pdf.

Lipnack, J., \& Stamps, J. (2000). Virtual teams: People working across boundaries with technology (2nd ed.). New York: Wiley.

Markus, M. L. (2005). Technology-shaping effects of E-Collaboration technologies: bugs and features. International Journal of $e$ Collaboration, 1(1), 1-23.

Orlikowski, W. J., \& Iacono, C. S. (2001). Research commentary: desperately seeking the "IT" in IT research — a call to theorizing the IT artifact. Information Systems Research, 12(2), 121-134.

Pipek, V., \& Wulf, V. (2009). Infrastructuring: towards an integrated perspective on the design and use of information technology.
Journal of the Association of Information Systems (JAIS), accepted for publication.

Powell, W. W. (1987). Hybrid organizational arrangements: new form or transitional development? California Management Review, 30, 67-87.

Prahalad, C. K., \& Hamel, G. (1990). The core competence of the corporation (HBS Reprint). In Harvard-Business-School (Ed.), Competitive strategy (pp. 11-24). Boston: HBS.

Quinn, J. B. (1992). Intelligent enterprise: A knowledge and service based paradigm for industry. New York: The Free.

Richter, A., \& Koch, M. (2008). The enterprise 2.0 story in Germany so far. Paper presented at the Workshop "What to expect from Enterprise 3.0: Adapting Web 2.0 to Corporate Reality" in International Conference on Computer-Supported Collaborative Work 2008.

Riemer, K. (2009). E-Collaboration systems - identification of system classes using cluster analysis. International Journal of $e$ Collaboration, 5(3), 1-24.

Riemer, K., \& Klein, S. (2006). Network management framework. In S. Klein \& A. Poulymenakou (Eds.), Managing dynamic networks (pp. 17-66). Berlin: Springer Verlag.

Riemer, K., \& Frößler, F. (2007). Introducing real-time collaboration systems: development of a conceptual scheme and research directions. Communications of the Association for Information Systems (CAIS), 20, 204-225.

Riemer, K., \& Vehring, N. (2008). E-Collaboration systems in virtual organizations-recommendations for tool support based on genre analysis. Paper presented at the 14th Americas Conference on Information Systems (AMCIS).

Riemer, K., \& Taing, S. (2009). Unified communications. Business \& Information Systems Engineering (BISE), 1(4), 326-330.

Riemer, K., Frößler, F., \& Klein, S. (2007). Real time communicationmodes of use in distributed teams. Paper presented at the 15th European conference on information systems, St.Gallen $(\mathrm{CH}), 07-$ 09 June 2007.

Saabeel, W., Verduijn, T., Hagdorn, L., \& Kumar, K. (2002). A model of virtual organisation: a structure and process perspective. eJoVElectronic Journal of Organizational Virtualness, 4(1), 1-17.

Schmalzl, B., Merkl, A., \& Imbery, H. (2004). Flexible office, desk sharing und hoteling - hotel service auch im Büro. In B. Schmalzl (Ed.), Arbeit und elektronische Kommunikation der Zukunft (pp. 265-284). Springer: Berlin et al.

Schmidt, K. (1991). Riding a tiger or computer supported cooperative work. In L. Bannon, M. Robinson \& K. Schmidt (Eds.), Proceedings of the second European conference on computer supported cooperative work. Dordrecht: Kluwer.

Schwabe, G. (2001). Koordinationswerkzeuge. In G. Schwabe, N. Streitz \& R. Unland (Eds.), CSCW-Kompendium (pp. 174-179). Springer: Berlin et al.

Sheth, J. N., \& Sharma, A. (1997). Supplier relationships - emerging issues and challenges. Industrial Marketing Management, 26, 91-100.

Teufel, S. (1996). Computerunterstützte Gruppenarbeit - eine Einführung. In H. Österle, \& P. Vogler (Eds.), Praxis des workflowmanagements (pp. 35-63). Braunschweig/Wiesbaden.

Teufel, S., Sauter, C., Mühlherr, T., \& Bauknecht, K. (1995). Computerunterstützung für die Gruppenarbeit. Bonn: Oldenbourg.

Wagner, M. P. (1995). Groupware und neues management. Einsatz geeigneter Softwaresysteme für flexiblere Organisationen. Braunschweig/Wiesbaden: Vieweg.

Weinkauf, K., Högl, M., \& Gemünden, H. G. (2004). Zusammenarbeit in innovativen Multi-Team-Projekten: Eine theoretische und empirische Analyse. Zeitschrift für betriebswirtschaftliche Forschung, 56, 419-435.

Wendel, T. (1996). Computerunterstützte Teamarbeit. Konzeption und Realisierung eines Team-arbeitssystems. Wiesbaden. 